\title{
Multiphase ISM in Nearby Early Type Galaxy IC 5063
}

\section{Bhagorao Tukaram Tate1,2, Anil Tejerao Kyadampure 2,3, Sheo Kumar Pandey, Madhav Khushalrao Patil ${ }^{*}$}

\author{
${ }^{1}$ Department of Physics, Balbhim Arts, Science and Commerce College, Beed, India \\ ${ }^{2}$ School of Physical Sciences, S. R. T. M. University, Nanded, India \\ ${ }^{3}$ Department of Physics, Sanjeevani Mahavidyalaya, Chapoli, India \\ ${ }^{4}$ School of Studies in Physics \& Astrophysics, Pt. R.S. University, Raipur, Chattisgarh, India \\ Email:*patil@iucaa.ernet.in
}

How to cite this paper: Tate, B.T., Kyadampure, A.T., Pandey, S.K. and Patil, M.K. (2018) Multiphase ISM in Nearby Early Type Galaxy IC 5063. International Journal of Astronomy and Astrophysics, 8, 79-93. https://doi.org/10.4236/ijaa.2018.81006

Received: January 7, 2018

Accepted: March 17, 2018

Published: March 20, 2018

Copyright (c) 2018 by authors and Scientific Research Publishing Inc. This work is licensed under the Creative Commons Attribution International License (CC BY 4.0).

http://creativecommons.org/licenses/by/4.0/

\begin{abstract}
A multiwavelength study of a nearby dust lane early-type galaxy IC 5063 is presented. The objectives are to investigate dust extinction properties and the association of interstellar dust with other phases of ISM. The color-index maps as well as the extinction maps derived from the analysis of deep CCD observations in optical passbands revealed a prominent dust lane along its optical major axis in the inner region. In addition, two more fainter and extended dust patterns are apparent in the color index map as well as extinction maps. These features are also evident in the smooth model subtracted residual maps. The extinction curve derived for this galaxy revealed that dust grains in it are identical to the canonical grains in the Milky Way with the dust grains little larger than the canonical grains. The total extinction measured in the $\mathrm{V}$ band extinction map enabled us to quantify the dust content of this galaxy to be equal to $4.9 \times 10^{4} M_{\odot}$, an order of magnitude shorter than that estimated using the IRAS flux densities at 60 and $100 \mu \mathrm{m}$. A Multiphase ISM study revealed a surprising similarity in the morphologies of the $\mathrm{H}_{\alpha}$ emitting ionized gas distribution and X-ray emitting gas. Systematic analysis of high resolution X-ray observations using Chandra telescope enabled us to detect 18 discrete X-ray sources within optical $\mathrm{D}_{25}$ region of IC 5063, out of which 17 sources were separated out as the low mass X-ray binaries and one as the high mass $\mathrm{X}$-ray binary source in the $\mathrm{X}$-ray color-color plot.
\end{abstract}

\section{Keywords}

Dust, Extinction, Galaxies: Individual (IC 5063), ISM 


\section{Introduction}

Classically, early-type galaxies (ETGs, both elliptical and lenticular collectively) were considered to be old, passive systems with a very low or almost devoid of gas and dust. However, with the availability of highly sensitive, state-of-the-art observing facilities covering wide range of electromagnetic spectrum have revealed that a notable fraction of these galaxies does host a substantial amount of interstellar medium (ISM) including interstellar dust. In fact, presence of dust and its systematic studies in this class of galaxies has acted as the theoretical smoking gun to trace the evolution and formation history of their host. Particularly, the diffusely distributed component of interstellar dust within galaxies has been chosen as the component to explore histories of ETGs due to its direct link with the cold gas in ISM. As a result, study of dust properties in this class of galaxies has been the topic of extensive study in last few decades.

A large fraction $(\sim 50 \%-80 \%)$ of ETGs, at least in the local universe, are now known to host interstellar dust in the form of lanes, filaments and patches, which has been confirmed through the careful analysis of high quality optical CCD imaging observations, thanks to the availability of sensitive array detectors [1] [2] [3] [4] [5]. These claims were further confirmed through the multiwavelength observations of these galaxies, particularly in the far-IR and sub-mm regions, investigating the presence of a substantial fraction of cold dust in these galaxies [6] [7]. X-ray observations of these galaxies have also detected a substantial amount of hot plasma heated to temperature about $10^{7} \mathrm{~K}$ in a large fraction of ETGs [3] [8] [9] [10]. Thus the presence of dust and ISM in various phases (atomic, molecular and plasma) is a common feature. The dust grains distributed within a galaxy modify the stellar spectral energy by absorbing or scattering photons of shorter wavelengths (UV and optical) and re-radiate it at longer wavelengths (FIR and sub-mm). The former is commonly referred to as the dust extinction and exhibit wavelength dependent nature, which can directly be envisaged in the form of dark lanes or patches in optical images. Therefore, systematic imaging analysis of a large sample of early-type galaxies is called for to understand the spatial distribution of dust in ETGs. The subsequent emission of the absorbed energy by the dust grains at longer wavelengths has been detected in a large sample of this class of galaxies by the Infra-Red Astronomical Satellite (IRAS) [6], the Infrared Space Observatory (ISO) and the Spitzer Space Telescope, and is also taken as the tracer for the current star formation in these galaxies [11]. IC 5063 is a potentially important candidate of Seyfert 2 type and has been studied extensively employing present day state-of-the-art multiwavelength observing facilities, including Chandra, HST, VLA, SPITZER, GALEX, etc. However, the main focus of these studies were to investigate AGN activity in at the core of this galaxy and its impact on the galaxy environemnt in general and ISM in particular. Further, a large attempt were made to explore the AGN jets and outflows [12] [13] [14].

The origin of dust in ETGs is one of the long debated issue. Three possible 
sources are supposed to yield the observed dust in ETGs: 1) formed in the atmospheres of the AGB stars, which then is shed into the ISM, 2) acquired through the merging or tidal capture from the neighbor, and 3) the same uncertain source as that in late-type galaxies e.g., growing continuously in the form of grains in ISM or supernovae, or left over from a vigorous star formation [15].

This paper presents dust extinction in a prominent dust lane early type galaxy IC 5063 (PKS 2048-57), which has been reported as a strong extended high-excitation optical line emitter and has also been detected as a powerful infrared emitter. The dust in this galaxy is found to extend out in the form of a lane till its edge [16] [17]. $\mathrm{H}_{\alpha}$ imaging has uncovered several bright knots of nebular emission lines within central 3" aperture as if they appear as multiple nuclei due to a merger event [18]. The global parameters of IC 5063 are given in Table 1.

The paper is organized as follows. Section 2 describes the optical and X-ray observations and data analysis processes, while Section 3 discusses the dust extinction properties and also compares its association with that of the ionized gas. Section 4 discusses results derived from the spatial and spectral analysis of $\mathrm{X}$-ray emission from this galaxy and also presents observed properties of discrete $\mathrm{X}$-ray binary sources that have been detected within the optical $\mathrm{D}_{25}$ region of this galaxy. The origin of dust and its association with other phases of ISM along with summary of the results are given in Section 5 .

Table 1. Globel parameters of IC 5063.

\begin{tabular}{cc}
\hline Parameter & Value \\
\hline Alternate names & PKS 2048-57, IRAS 20481-5715, PGC 065600 \\
ESO 204812-5715.5 & $20: 52: 02.3 ;-57: 04: 08$ \\
RA DEC & SAO \\
Morphology & 12.89 \\
Mag BT & $2^{\prime} .1 \times 1^{\prime} .4$ \\
Dimensions & $12^{\prime} \times 8^{\prime} 5$ \\
D25 & 45.3 \\
Distance (Mpc) & 0.01114 \\
Redshift (z) & $3402 \pm 6$ \\
Radial velocity (km/s) & $1.290 \pm 0.024(12 \mu \mathrm{m}) ; 4.270 \pm 0.030(24 \mu \mathrm{m})$ \\
IRAS flux density (Jy) & $5.87 \pm 0.038(60 \mu \mathrm{m}) ; 4.25 \pm 0.207(100 \mu \mathrm{m})$ \\
& $408 \mathrm{MHz}: 6.200 \mathrm{Jy}$ \\
Radio flux density &
\end{tabular}




\section{Data Reduction}

\subsection{Optical Data}

Deep, high S/N CCD images in broad band filters B, V, R, I and narrow band filter $\mathrm{H}_{\alpha}$ on this dust lane galaxy IC 5063 observed with Danish 1.54-metre telescope with Instrument Adapter-No.17 at La Silla operated by ESO (European Southern observatory) were acquired from NED [19]. These images were bias subtracted, flat fielded and co-added to achieve good S/N. We processed these images following standard data analysis tasks within IRAF. The sky background was estimated employing the box method and was then subtracted from the respective science images. All the five images were then aligned geometrically employing the task geomap and geotran within IRAF. We also acquired near-IR J, H and Ks band images of IC 5063 from the archive of the Two Micron All Sky Survey (2MASS) and near UV and far-UV images from the GALEX archive.

\subsection{X-Ray Data}

Though X-ray emission properties of IC 5063 using Chandra observations have already been reported by LaMassa (2011) and LaMassa (2012) [20] [21], systematic comparison of the morphologies of the multiphase ISM in the merger remnant galaxy has not yet been attempted. For delineating the X-ray emission from this galaxy we have utilized high-resolution data available in the archive of Chandra observatory. IC 5063 was observed by Evans (2006) [22] with Chandra on 20070615 (Obs. ID 7878) positioned at the center of ACIS-S3 for an effective exposure of $34.1 \mathrm{ks}$. We retrieved the level-1 event files corresponding to this ObsID and processed it adopting the standard data preparation tasks within Chandra Interactive Analysis of Observations (CIAO version 4.6.7) and recent calibration files provided by the Chandra X-ray Center (CXC) (CALDB version 4.7.7). Bad pixels and other artefacts were identified and removed from the level-1 data sets by running the script chandra_repro. The background light curve was derived by extracting $2-7 \mathrm{keV} \mathrm{X}$-ray photons from chip S3 away from the source and the periods of high background flaring events (with $3 \sigma$ clipping threshold) were identified and removed from the data sets using the script Ic_sigma_clip within SHERPA [23]. Removal of such flaring segments from the data reduced the effective exposure of the observation to $34.1 \mathrm{ks}$. We used ACIS-S blank-sky observations provided by the Chandra X-ray centre (CXC) for sky background subtraction from the science data. CIAO task acis_bkgrnd lookup was used to identify the blank-sky data corresponding to IC 5063 observations and the background count rates in 9-12 keV band were adjusted so as to match those in the source image [24]. As IC 5063 was observed in Very Faint (VFAINT) telemetry format, therefore, we applied VFAINT background screening to the blank-sky file after including events with status $=0$. Exposure map and exposure-corrected image of IC 5063 in $0.3-3 \mathrm{keV}$ energy band were created using standard CIAO FLUXIMAGE script. Point sources across the field 
of view were detected through the CIAO script wavdetect [23] and were excluded while deriving X-ray emission map of IC 5063. For investigating thermodynamical properties of the X-ray emitting gas from regions of interest from within IC 5063 we extracted the source spectra, background spectra, response and effective area files using task specextract within CIAO, which were then fitted using spectral analysis package XSPEC-12.8.1.

\section{Results and Discussion}

\subsection{Dust Properties}

Though the presence of interstellar dust in IC 5063 has been known since long time [17] [25] quantitative measurements of the wavelength dependent nature of dust extinction in this galaxy were not available in the literature. The extent of dust extinction and its wavelength dependence is a strong function of the size distribution, structure and chemical composition of the dust grains. As a first step in delineating the dust extinction nature we investigated the actual extent and spatial distribution of dust in this galaxy by deriving its color index maps $(\mathrm{B}-\mathrm{V}),(\mathrm{B}-\mathrm{R})$, etc., which requires comparison of the light distribution in the geometrically aligned, seeing matched broad band $B, V, R, I$ images. Figure 1 shows one of such a color-index maps (B-R) of IC 5063, the brighter shades in which reveals the dust occupied regions, matching well with that reported by Colina (1991) [26].

Once morphology of the dust was confirmed in the color index maps, next step was to quantify the dust extinction in each of the passband. This require comparing the stellar light distribution in the presence and absence of interstellar dust in the target galaxy i.e., by comparing light distribution in the cleaned science frame with that of its 2D smooth model [27]. For this we obtained dust free models of IC 5063 in each of the passband by employing two different approaches. In the first attempt we fit isophotes of light distribution in this galaxy with ellipses using the ISOPHOTE package within IRAF (see Patil et al., 2007 [3] for details). We allowed the position angle, ellipticity of each of the isophote to vary, while the center coordinates were fixed during the fit. The fitting was continued till the signal reaches $3 \sigma$ of the background. Before this all the obvious regions occupying dust and foreground stars were masked and ignored during the fit. Dust free models of IC 5063 in each of the passband were also obtained employing the GALFIT 3.0.5 code of [28].

This code uses the Sersic model [29] for deriving light distribution within the program galaxies. We provided the proper image size, the point spread function (psf) of each of the band pass, initial guess parameters like, position angle, ellipticity, center coordinates as input to the GALFIT. Here too, we masked the foreground stars while deriving the two dimensional smooth models so that GALFIT yields a better fit with the reduced $\chi^{2}$ [28]. We then derived the residual maps of IC 5063 after subtracting dust free models from original science frames. The $\mathrm{V}$ and $\mathrm{R}$ band residual maps thus derived are shown in Figure 2. 


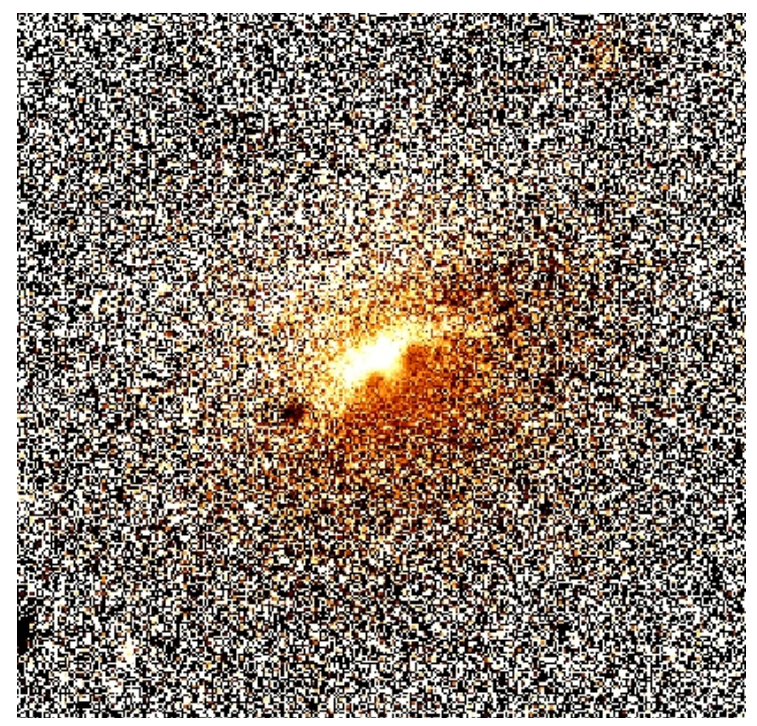

Figure 1. (B-R) color index map of IC 5063 delineating dust occupied region (brighter shades).

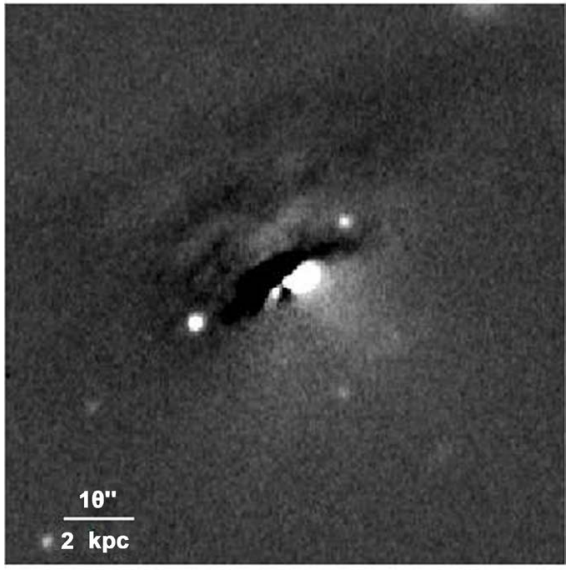

(a)

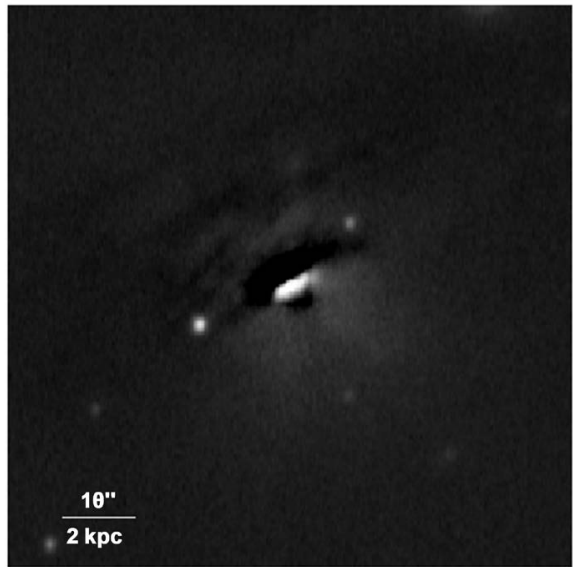

(b)

Figure 2. Residual maps in $\mathrm{V}$ and $\mathrm{R}$ bands (left and right panels, respectively) derived for IC 5063 after subtracting 2D smooth models derived with GALFIT. Darker shades in both the panels exhibit dust occupied region.

Dust free models thus generated were then used to quantify the wavelength dependent nature of the dust extinction, the extinction curve, using relation

$$
A_{\lambda}=-2.5 \log \left(\frac{I_{\lambda, \text { obs }}}{I_{\lambda, \text { model }}}\right)
$$

where, $A_{\lambda}$ gives the amount of total extinction in a particular pass band $(B, V, R, I)$ measured in magnitude scale, while $I_{\lambda, \text { obs }}$ and $I_{\lambda \text {,model }}$ represent the observed (attenuated) and un-attenuated light intensities in a given pass band, respectively. Two of such extinction maps in V and R band for IC 5063 are shown in Figure 3, where the brighter shades corresponds to the dust extinguished regions. We then investigated the quantitative dependence of the dust extinction and its 


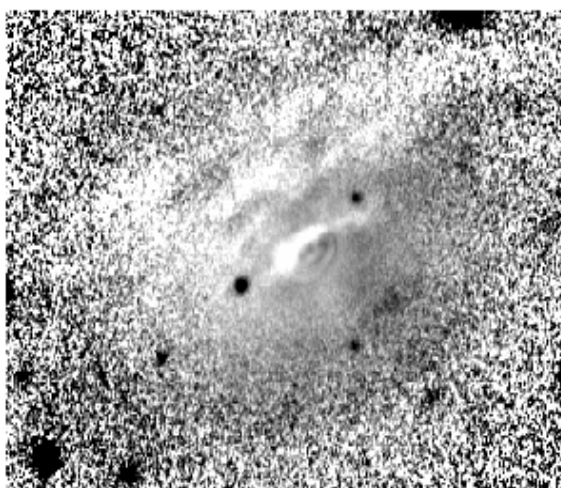

(a)

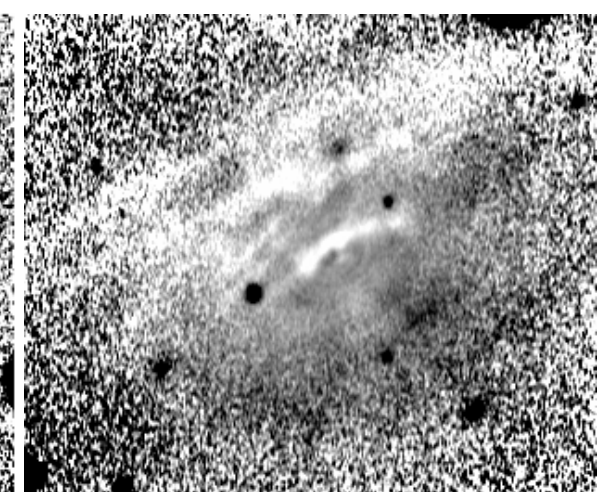

(b)

Figure 3. Exinction maps of IC 5063 in V and R bands derived after dividing the cleaned $\mathrm{V}$ and $\mathrm{R}$ band images by their respective $2 \mathrm{D}$ smooth models using ellipse fitting. The brighter shades in both the panels exhibit the dust extinguished regions.

wavelength dependent nature by measuring total extinction values by sliding a $5 \times 5$ box over the dust-extinguished regions. The numerical values of local extinctions in each of the passband $\left(A_{\lambda}\right)$ measured using box method were then used to derive the extinctions $\left(R_{\lambda}\right)$ by fitting linear regressions to the total extinction $A_{\lambda}$ versus the selective extinction $E(B-V)=A_{B}-A_{V}$. Slopes of the best regression fits along with their associated uncertainties yielded the $R_{\lambda}$ and hence the extinction curve. The extinction curve, the graph between the measured values of $R_{\lambda}\left(=\frac{A_{\lambda}}{E(B-V)}\right)$ versus inverse of the wavelength, for IC 5063 is shown in Figure 4 and is found to exhibit linear relation. For comparison we also plot a similar curve for the Milky Way (shown in red line, [30]) in the same plot. The extinction curve for the program galaxy is found to run parallel to that for the canonical curve indicating that the dust extinction in both of the galaxies is of identical nature. Similar inferences were also arrived at for a number of early-type galaxies [2] [3] [9] [10] [31] [32] [33]. However, the dust grain size for that in IC 5063 appears to be little larger than the canonical grains in the Milky Way with the average grain size equal to $\left\langle\frac{a}{a_{G a l}}\right\rangle=1.008 \pm 0.08$ and $R_{V}$ equal to $4.02 \pm 0.25$.

\subsubsection{Dust Content of IC 5063}

Total dust content of IC 5063 was estimated using the total extinction values measured from within the $\mathrm{V}$ band extinction map. For this we integrate the dust column density $\Sigma_{d}$ over the dust occupied area $(A)$. Here, we assume that the dust grains within IC 5063 have chemical composition and geometry similar to that of the canonical grains in the Milky Way. Assuming spherical grains of Mathis et al. (1977) [30] size distribution of silicate and graphite uncoated refractory particles we quantify the total dust content of this galaxy using relation 


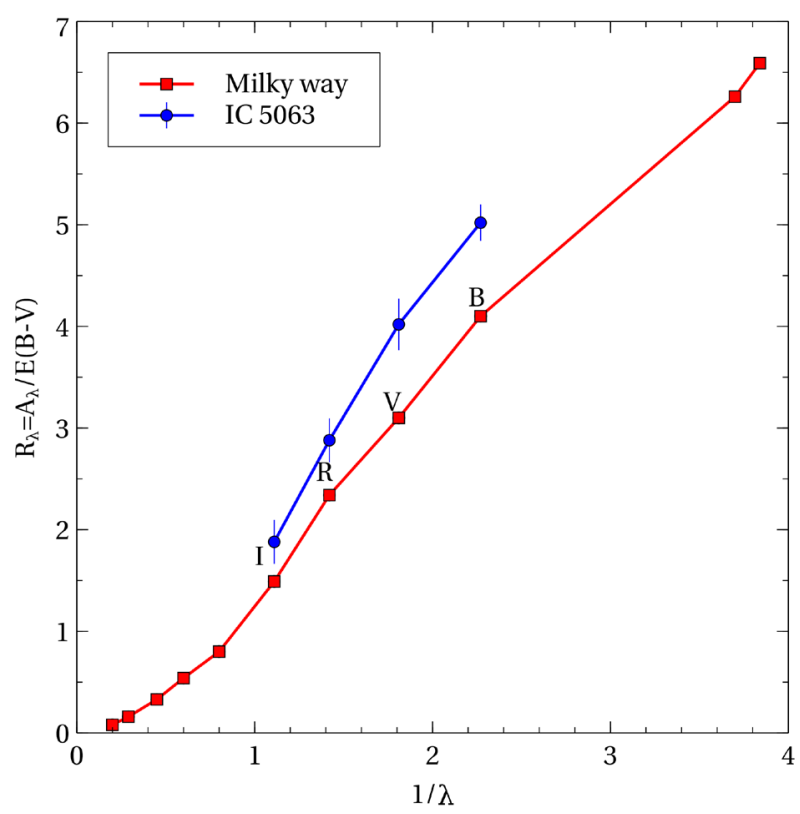

Figure 4. The extinction curve for IC 5063 (blue line) relative to that for the canonical grains in the Milky Way (red line).

$$
M_{d}=A \times \Sigma_{d}=A \times l_{d} \times \int_{a_{\min }}^{a_{\max }} \frac{4}{3} \pi a^{3} \rho_{d} n(a) \mathrm{d} a
$$

where, $n(a) \mathrm{d} a\left(=n_{H} A_{i} a^{-3.5} \mathrm{~d} a\right)$ gives particle size distribution of $a, A_{i}$ the overall abundance of silicate and graphite grains, $n_{H}$ the hydrogen number density; $\Sigma_{d}$ the dust column density; $\rho_{d}$ the specific grain size density $(\sim 3 \mathrm{~g}$ $\left.\mathrm{cm}^{-3}\right) ; l_{d}$ the dust column length along the line of sight; and $a_{\max }$ and $a_{\min }$ representing the upper and lower cutoffs of the size distribution, respectively. The measured values of the average extinction in $\mathrm{V}$ band $\left(\left\langle A_{V}\right\rangle\right)$ yielded the dust content of IC 5063 to be equal to $4.9 \times 10^{4} M_{\odot}$. However, this estimate provides the lower limit due to the reason that this method is based on the screening effect of dust grains. The grains that are distributed throughout the galaxy would not be accounted for in this method. This estimate is further affected by the uncertainties due to the lower and upper cutoffs of the grain sizes.

An alternative attempt was also made to quantify the true dust content of IC 5063 employing the far-IR emission flux densities of interstellar dust grains at 60 $\mu \mathrm{m}$ and $100 \mu \mathrm{m}$ measured by the IRAS satellite. As a first step in this attempt, we derive the dust grain temperature using relation $T_{d}=49\left(\frac{S_{60}}{S_{100}}\right)^{0.4} \mathrm{~K} \quad$ [34], which was found to be $58 \mathrm{~K}$, hot relative to the grains evidenced in other dusty early-type galaxies [3]. Then we estimate the dust mass from measured values of IRAS flux densities to be equal to $4.5 \times 10^{5} M_{\odot}$ [35], an order of magnitude higher than the estimate from optical extinction method and is consistent with the findings by several other researchers [3] [31] [36]. This discrepancy in the two estimates is because the diffuse component of dust that could not get 
detected in the optical method has contributed substantially in the measured values of IRAS flux densities.

\subsection{X-Ray Emission}

With an analogy to examine morphology of the X-ray emitting hot gas and also to investigate its association with that of the interstellar dust we have derived X-ray emission maps of IC 5063 employing 34.1 ks Chandra observations. A 0.3 - $3 \mathrm{keV}$ background subtracted, exposure corrected, point source removed, $3 \sigma$ smoothed X-ray emission map for IC 5063 is shown in Figure 5(a). In the same figure we also overlay contours of dust occupied region, which exhibit that the $\mathrm{X}$-ray emitting flux is diminished along the dust occupied region revealing that $\mathrm{X}$-rays are lost along this region. We also derive $\mathrm{X}$-ray tri-color map by combining $\mathrm{X}$-ray photons extracted in three different energy bands namely, the soft (0.3 - 1 $\mathrm{keV}$, shown in red color), medium ( $1-2 \mathrm{keV}$, green color) and hard (2 - $7 \mathrm{keV}$, blue color). The resultant X-ray tri-color map is shown in Figure 5(b), which indicate that the $\mathrm{X}$-ray emission in the central region is dominated by harder component while the diffuse emission is mostly due to the soft component.

Global properties of the X-ray emitting gas from within IC 5063 were also investigated by extracting a $0.3-7 \mathrm{keV}$ combined spectrum of the X-ray photons from within its optical $\mathrm{D}_{25}$ region. Emission from central 1 "was excluded from this analysis to avoid the harder component originating from the nuclear source. The spectrum was then imported to XSPEC $\mathrm{v}$ 12.7.1 and was fitted with an absorbed single temperature MEKAL model following the standard $\chi^{2}$ statistics and fixing the hydrogen column density at the Galactic value of $6.4 \times 10^{21} \mathrm{~cm}^{-2}$ ) [20]. However, the fit yielded large residuals particularly in the higher energy range, therefore, we added power law component to it. This resulted in a relatively better fit with $\chi^{2}$ close to 1.03 and the best-fit temperature $\mathrm{kT}=0.28 \pm$ 0.05 and photon index $\Gamma=1.05 \pm 0.17$.

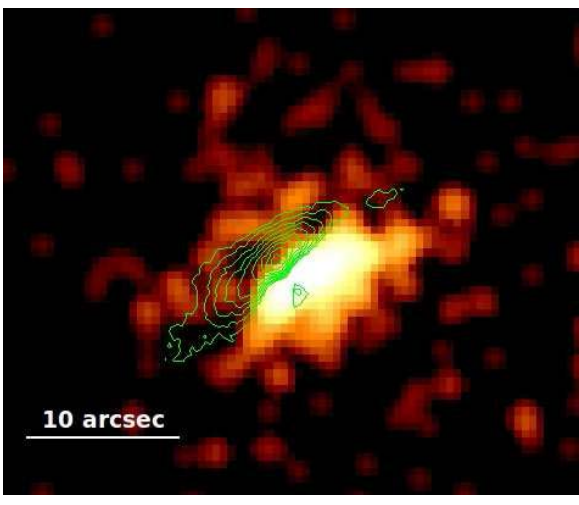

(a)

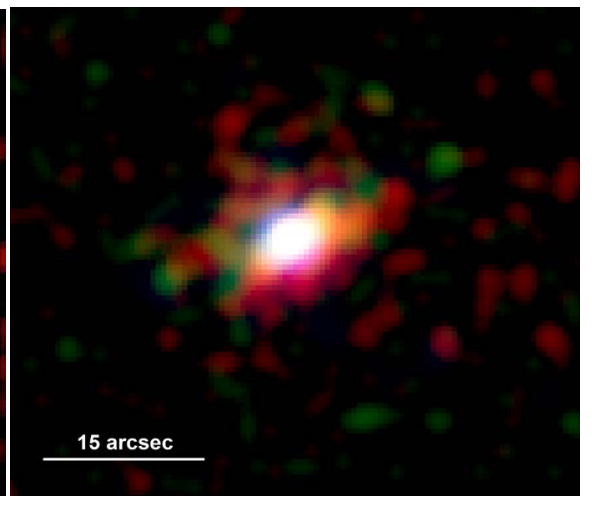

(b)

Figure 5. (a): Morphology of the X-ray emitting gas distribution within IC 5063 derived from the analysis of deep X-ray data from Chandra observatory. The green contours on this figure indicates the dust occupied region, indicating that X-ray photons in the dust occupied regions are extinguished substantially. (b): X-ray tri-color map delineating different components of X-ray emitting gas. 


\subsection{X-Ray Binary Sources}

X-ray study of this galaxy enabled us to resolve a set of 18 discrete X-ray binary sources (XRBs) within optical $\mathrm{D}_{25}$ region of IC 5063 of the Chandra image. XRBs are believed to be associated with the star forming history and hence act as proxy to investigate formation scenario of the host galaxy [37].

As a result several attempts were made in the past to investigate population of these sources in early-type galaxies using the high spatial resolution data from Chandra observatory [23] [38]. An XRB has a dwarf companion like neutron star or a black hole that accretes matter from a gravitationally bound evolved star, accretion of which yields in X-ray emission. Therefore, to investigate the characteristics of the dwarf companion it is required to understand its spectral nature. However, spectral analysis of X-ray emission from such discrete sources is not always possible due to insufficient statistics in majority of the sources. Alternatively we employed the X-ray color plots of the discrete sources to investigate their spectral properties and also to classify the resolved sources in different types [39].

For this purpose following Vagshette et al. (2013) [23] we extract background subtracted X-ray counts from individual source in three different energy bands namely, the soft (S, $0.3-1 \mathrm{keV})$, the medium (M, $1-2 \mathrm{keV})$ and the hard $(\mathrm{H}, 2$ $7 \mathrm{keV}$ ) using task dmextract within CIAO. Then we estimated the hardness ratios as X21 $=(\mathrm{M}-\mathrm{S}) /(\mathrm{S}+\mathrm{M}+\mathrm{H}), \mathrm{X} 31=(\mathrm{H}-\mathrm{S}) /(\mathrm{S}+\mathrm{M}+\mathrm{H})$, where $\mathrm{S}, \mathrm{M}$ and $\mathrm{H}$ represent the $\mathrm{X}$-ray counts in the soft, medium and hard bands, respectively.

The plot between the measured values of X-ray hardness ratios X31 versus the soft color X21 is shown in Figure 6, which clearly reveals that out of the resolved

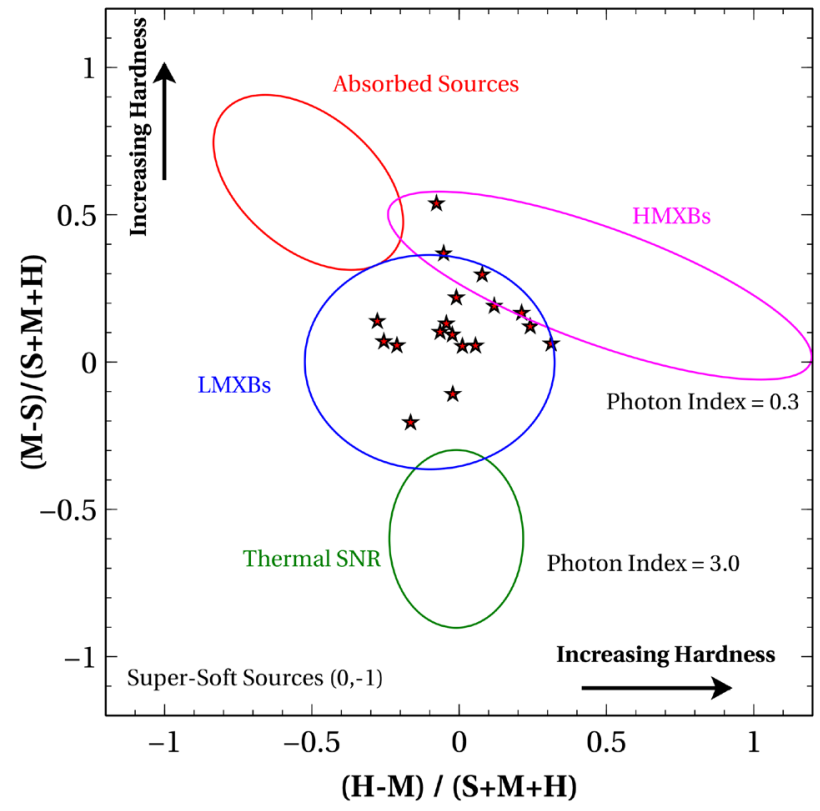

Figure 6. Shows the Hardness ratio of $\mathrm{X}$-ray point sources in IC 5063. The hardness ratios defined as $\mathrm{X} 21=(\mathrm{M}-\mathrm{S}) /(\mathrm{S}+\mathrm{M}+\mathrm{H})$ and $\mathrm{X} 31=(\mathrm{H}-\mathrm{M}) /(\mathrm{S}+\mathrm{M}+\mathrm{H})$. 
18 discrete sources 17 belongs to the region classified as low mass X-ray binaries (LMXBs), while only one source was found to be of high mass X-ray binary (HMXB). This analysis failed to detect any of the super-soft as well as AGN source in the field.

\subsection{Multiphase ISM}

With an objective to examine the association of dust with ionized gas, we have derived $\mathrm{H}_{\alpha}+[\mathrm{N}$ II] emission map of IC 5063. Spatial distribution of the ionized gas within IC 5063 is shown in Figure 7(a), which closely matches with that of the dust evident in (B-R) colour map as well as X-ray emission map in $0.3-3.0$ $\mathrm{keV}$. The $\mathrm{H}_{\alpha}$ emission map also reveals the filamentary structures of emission nebulosity. Figure 7(b) indicates the spatial correspondence of dust with the ionized gas. This was also confirmed in the tricolor map (Figure 7 (a)) produced after combining images in three different bands i.e. optical $\mathrm{V}$ band from HST (shown in red), ionized gas morphology centered on $\mathrm{H}_{\alpha}$ emission (shown in green) and 0.3 - $3 \mathrm{keV}$ X-ray emission mapped by Chandra telescope (in blue). This figure clearly endorses the morphological similarities of the X-ray emitting gas with that of the ionized gas. Morphological similarities of the three components indicate that all the three components have a common origin. Thus, the systematic multiwavelength study of IC 5063 allows us to examine the spatial correspondence between the interstellar dust, warm ionized gas and X-ray hot gas.

\section{Conclusions}

This paper presents multiwavelength study of a dust lane early-type galaxy IC 5063 with an objective to investigate dust extinction properties and association of dust with other phases of ISM. The main results from this study are

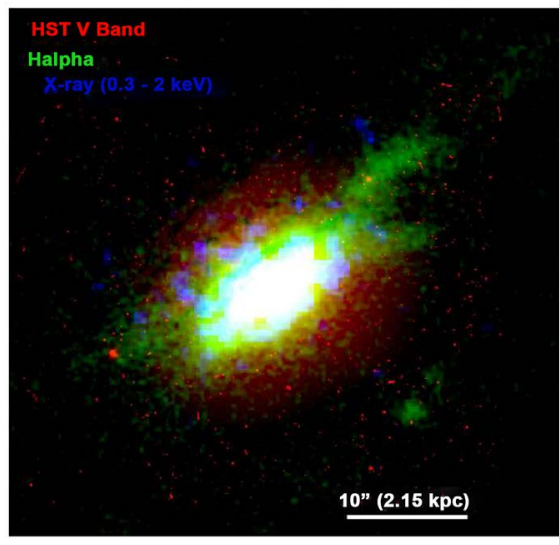

(a)

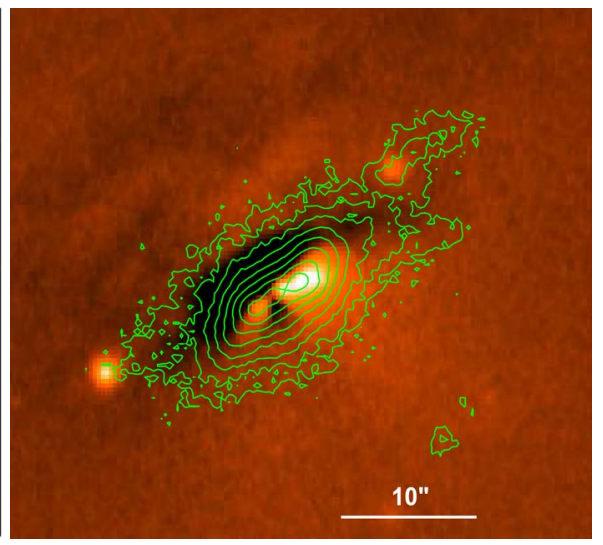

(b)

Figure 7. (a): Tricolor map delineating multiphase ISM association in IC 5063. Red color in it represents the contribution from stellar light mapped through the HST V band image, green color representing the morphology of the ionized gas distribution centered on $\mathrm{H}_{\alpha}$ emission, while blue color represents the $0.3-2 \mathrm{keV}$ X-ray emission mapped through deep observations by Chandra telescope; (b): $\mathrm{H}_{\alpha}$ emission contours overlaid on the dust extinction region (darker shades) in $\mathrm{V}$ band. 
summarized below.

- Imaging analysis of deep CCD observations of IC 5063 in optical passbands reveal a prominent dust lane along its optical major axis in the inner region. In addition to the prominent dust lane two more relatively fainter and extended dust patterns are apparent in color index map as well as extinction maps. These features were also evident in smooth model subtracted residual maps.

- To delineate the extinction characteristics of dust grains within IC 5063 we obtain the extinction curve as a function of wavelength by deriving its extinction maps in different passbands. Comparison of this curve with that derived for the canonical grain in the Milky Way reveals that the dust grains in the target galaxy exhibit identical characteristics.

- The total extinction measured in the $\mathrm{V}$ band extinction map enabled us to quantify the dust content of this galaxy to be equal to $4.9 \times 10^{4} M_{\odot}$, which was an order of magnitude shorter than that estimated using the IRAS flux densities at 60 and $100 \mu \mathrm{m}$.

- With an objective to investigate the presence of other phases of ISM in this galaxy we have also derived its nebular emission maps centered on the $\mathrm{H}_{\alpha}$ emission and X-ray emission maps. A surprising similarity was evidenced in the morphologies of the $\mathrm{H}_{\alpha}$ emitting ionized gas distribution and X-ray emitting gas.

- High resolution X-ray imaging analysis of Chandra observations of this galaxy also enabled us to detect 18 discrete X-ray binary sources within optical $\mathrm{D}_{25}$ region of IC 5063, out of which 17 sources were separated out as the low mass X-ray binaries and one as the high mass X-ray binary source in the X-ray color-color plot.

\section{Acknowledgements}

We are thankful to the referee for their valuable suggestions and comments to improve content of this paper. This work has made use of data from the NASA/IPAC Extragalactic Database (NED), which is operated by the Jet Propulsion Laboratory, California Institute of Technology, under contract with the National Aeronautics and Space Administration. This publication has made use of data products from the Chandra, ESO (Danish), GALEX, 2MASS and IRAC archives.

\section{References}

[1] Goudfrooij, P., de Jong, T., Hansen, L. and Norgaard-Nielsen, H.U. (1994) Interstellar Matter in Elliptical Galaxies-Part Three-Properties of Dust Extinction. MNRAS, 271, 833-851. https://doi.org/10.1093/mnras/271.4.833

[2] Patil, M.K., Pandey, S.K., Sahu, D.K. and Kembhavi, A.K. (2003) Multi-Wavelength Study of Dust Properties in Early-Type Galaxies. In: Astrophysics of Dust, Estes Park, Colorado, 26-30 May 2003.

[3] Patil, M.K., Pandey, S.K., Sahu, D.K. and Kembhavi, A. (2007) Properties of Dust in 
Early-Type Galaxies. $A \& A, 461,103-113$.

[4] Deshmukh, S.P., Tate, B.T., Vagshette, N.D., Pandey, S.K. and Patil, M.K. (2013) A Multiwavelength View of the ISM in the Merger Remnant Galaxy Fornax A. Research in Astronomy and Astrophysics, 13, 885-898.

https://doi.org/10.1088/1674-4527/13/8/001

[5] Kulkarni, S., Sahu, D.K., Chaware, L., Chakradhari, N.K. and Pandey, S.K. (2014) Study of Dust and Ionized Gas in Early-Type Galaxies. New Astronomy, 30, 51-63. https://doi.org/10.1016/j.newast.2014.01.003

[6] Knapp, G.R., Guhathakurta, P., Kim, D.-W. and Jura, M.A. (1989) Interstellar Matter in Early-Type Galaxies. I-IRAS Flux Densities. Astrophysical Journal Supplement Series, 70, 329-387. https://doi.org/10.1086/191342

[7] Goudfrooij, P. and de Jong, T. (1995) Interstellar Matter in Shapley-Ames Elliptical Galaxies. IV. A Diffusely Distributed Component of Dust and Its Effect on Colour Gradients. $A \& A, 298,784$.

[8] Kim, D.-W., Fabbiano, G. and Trinchieri, G. (1992) The X-Ray Spectra of Galaxies. II-Average Spectral Properties and Emission Mechanisms. The Astrophysical Journal, 393, 134-148. https://doi.org/10.1086/171492

[9] Vagshette, N.D., Pandge, M.B., Pandey, S.K. and Patil, M.K. (2012) Dust Extinction and X-Ray Emission from the Starburst Galaxy NGC 1482. New Astronomy, 17, 524-532. https://doi.org/10.1016/j.newast.2011.12.005

[10] Patil, M.K., Pandey, S.K., Kembhavi, A. and Sahu, D.K. (2009) Multiband Imaging Observations of a Prominent Dust Lane Galaxy NGC 4370. Astronomy \& Astrophysics Manuscript No. ngc4370.

[11] Leeuw, L.L., Sansom, A.E., Robson, E.I., Haas, M. and Kuno, N. (2004) Observations of Cold Dust in Nearby Elliptical Galaxies. The Astrophysical Journal, 612, 837-847. https://doi.org/10.1086/422795

[12] Tadhunter, C., Morganti, R., Rose, M., Oonk, J.B.R. and Oosterloo, T. (2014) Jet Acceleration of the Fast Molecular Outflows in the Seyfert Galaxy IC 5063. Nature, 511, 440-443. https://doi.org/10.1038/nature13520

[13] Morganti, R., Frieswijk, W., Oonk, R.J.B., Oosterloo, T. and Tadhunter, C. (2013) Tracing the Extreme Interplay between Radio Jets and the ISM in IC 5063. $A \& A$, $552, \mathrm{~L} 4$.

[14] Congiu, E., Contini, M., Ciroi, S., Cracco, V., Berton, M., Di Mille, F., Frezzato, M., La Mura, G. and Rafanelli, P. (2017) High-Resolution Spectroscopy of the Extended Narrow-Line Region of IC 5063 and NGC 7212. Monthly Notices of the Royal Astronomical Society, 471, 562-588. https://doi.org/10.1093/mnras/stx1628

[15] Morgan, H.L. and Edmunds, M.G. (2003) Dust Formation in Early Galaxies. Monthly Notices of the Royal Astronomical Society, 343, 427-442. https://doi.org/10.1046/j.1365-8711.2003.06681.x

[16] Danziger, I.J., Goss, W.M. and Wellington, K.J. (1981) The Dynamics of the S0 Galaxy IC 5063. Monthly Notices of the Royal Astronomical Society, 196, 845-856. https://doi.org/10.1093/mnras/196.4.845

[17] Colina, L., Sparks, W.B. and Macchetto, F. (1992) IC 5063: A Merger Remnant with a Hidden Luminous Active Nucleus: Erratum. The Astrophysical Journal, 385, 766. https://doi.org/10.1086/170984

[18] Heisler, C.A., Vader, J.P. and Frogel, J.A. (1989) The Double-Nucleus Galaxy IRAS 02580-1136-A Merging System. The Astrophysical Journal, 97, 986-994.

[19] Chatzichristou, E.T. (2000) Multicolor Optical Imaging of Infrared-Warm Seyfert 
Galaxies. I. Introduction and Sample Selection. The Astrophysical Journal, 131, 71-94. https://doi.org/10.1086/317362

[20] LaMassa, S.M. (2011) Uncovering Hidden Black Holes: Obscured AGN and Their Relationship to the Host Galaxy. PhD Thesis, The Johns Hopkins University, Baltimore.

[21] LaMassa, S.M., Heckman, T.M. and Ptak, A. (2012) Disentangling AGN and Star Formation in Soft X-Rays. The Astrophysical Journal, 758, 82. https://doi.org/10.1088/0004-637X/758/2/82

[22] Evans, D. (2006) The Circumnuclear X-Ray Environments of Highly Disturbed Seyfert Galaxies.

[23] Vagshette, N.D., Pandge, M.B. and Patil, M.K. (2013) Spectral Properties of XRBs in Dusty Early-Type Galaxies. New Astronomy, 21, 1-7. https://doi.org/10.1016/j.newast.2012.10.005

[24] Markevitch, M., Gonzalez, A.H., David, L., Vikhlinin, A., Murray, S., Forman, W., Jones, C. and Tucker, W. (2002) A Textbook Example of a Bow Shock in the Merging Galaxy Cluster 1E 0657-56. The Astrophysical Journal, 567, L27-L31. https://doi.org/10.1086/339619

[25] Schweizer, F. (1980) An Optical Study of the Giant Radio Galaxy NGC 1316/Fornax A/. The Astrophysical Journal, 237, 303-318. https://doi.org/10.1086/157870

[26] Colina, L., Sparks, W.B. and Macchetto, F. (1991) IC 5063-A Merger Remnant with a Hidden Luminous Active Nucleus. The Astrophysical Journal, 370, 102-117. https://doi.org/10.1086/169795

[27] Brosch, N. (1987) Extragalactic Dust. II Far-Infrared Properties of Early-Type Galaxies with Dust Lanes. Monthly Notices of the Royal Astronomical Society, 225, 257-266. https://doi.org/10.1093/mnras/225.2.257

[28] Peng, C.Y., Ho, L.C., Impey, C.D. and Rix, H.-W. (2002) Detailed Structural Decomposition of Galaxy Images. The Astrophysical Journal, 124, 266-293.

[29] Caon, N., Capaccioli, M. and D’Onofrio, M. (1993) On the Shape of the Light Profiles of Early Type Galaxies. Monthly Notices of the Royal Astronomical Society, 265, 1013-1021. https://doi.org/10.1093/mnras/265.4.1013

[30] Mathis, J.S., Rumpl, W. and Nordsieck, K.H. (1977) The Size Distribution of Interstellar Grains. The Astrophysical Journal, 217, 425-433. https://doi.org/10.1086/155591

[31] Goudfrooij, P., Hansen, L., Jorgensen, H.E. and Norgaard-Nielsen, H.U. (1994) Interstellar Matter in Shapley-Ames Elliptical Galaxies. II. The Distribution of Dust and Ionized Gas. Astronomy and Astrophysics Supplement Series, 105, 341-383.

[32] Patil, M.K., Pandey, S.K., Kembhavi, A.K. and Singh, M. (2001) Dust in an Extragalactic Environment: The Case of NGC 2907. Bulletin of the Astronomical Society of India, 29, 453-455.

[33] Finkelman, I., Brosch, N., Kniazev, A.Y., Väisänen, P., Buckley, D.A.H., O’Donoghue, D., Gulbis, A., Hashimoto, Y., Loaring, N., Romero-Colmenero, E. and Sefako, R. (2010) Determining the Extragalactic Extinction Law with SALT. II. Additional Sample. Monthly Notices of the Royal Astronomical Society, 409, 727-736. https://doi.org/10.1111/j.1365-2966.2010.17334.x

[34] Young, J.S., Xie, S., Kenney, J.D.P. and Rice, W.L. (1989) Global Properties of Infrared Bright Galaxies. The Astrophysical Journal, 70, 699-722. https://doi.org/10.1086/191355

[35] Hildebrand, R.H. (1983) The Determination of Cloud Masses and Dust Characteris- 
tics from Submillimetre Thermal Emission. Quarterly Journal of the Royal Astronomical Society, 24, 267-282.

[36] Finkelman, I., Brosch, N., Funes, J.G., Barway, S., Kniazev, A. and Väisänen, P. (2012) Dust and Ionized Gas Association in E/S0 Galaxies with Dust Lanes: Clues to Their Origin. Monthly Notices of the Royal Astronomical Society, 422, 1384-1393. https://doi.org/10.1111/j.1365-2966.2012.20710.x

[37] Irwin, J.A., Athey, A.E. and Bregman, J.N. (2003) X-Ray Spectral Properties of Low-Mass X-Ray Binaries in Nearby Galaxies. The Astrophysical Journal, 587, 356-366. https://doi.org/10.1086/368179

[38] Colbert, E.J.M., Heckman, T.M., Ptak, A.F., Strickland, D.K. and Weaver, K.A. (2004) Old and Young X-Ray Point Source Populations in Nearby Galaxies. The Astrophysical Journal, 602, 231-248. https://doi.org/10.1086/380899

[39] Fabbiano, G., Kim, D.-W., Fragos, T., Kalogera, V., King, A.R., Angelini, L., Davies, R.L., Gallagher, J.S., Pellegrini, S., Trinchieri, G., Zepf, S.E. and Zezas, A. (2006) The Modulated Emission of the Ultraluminous X-Ray Source in NGC 3379. The Astrophysical Journal, 650, 879-884. https://doi.org/10.1086/507018 